\title{
Research on Industrial Robot Monitoring Technology Based on PC SDK
}

\author{
Xie Kunpeng \\ Tianjin University of Technology and Education \\ Institute of robotics and intelligent equipment \\ Tianjin, China \\ Qi Yuming \\ Tianjin University of Technology and Education \\ Institute of robotics and intelligent equipment \\ Tianjin, China
}

\author{
Liu Chaohua \\ Tianjin University of Technology and Education \\ Institute of robotics and intelligent equipment \\ Tianjin, China \\ Zhou Hongchao \\ Tianjin University of Technology and Education \\ Institute of robotics and intelligent equipment \\ Tianjin, China
}

\begin{abstract}
Around the ABB Industrial robot monitoring technology problem, study elaborated the composition and the application function of PC SDK development Interface Class Library based on Net platform, compared and analyzed the advantages and disadvantages of two development environment (virtual environment and real environment) of the robot Monitoring system under PC SDK. Besides, programmed a ABB robot local multi-machine monitoring system using $C$ \# language in the virtual development environment. Finally, connected the virtual controller, and verified the usability of the monitoring system. Some functions of the PC SDK were also realized.
\end{abstract}

\section{Keywords-Industrial Robot, PC SDK, C\#, Monitoring System}

\section{INTRODUCTION}

With the rapid development of modern industry, industrial robots are utilized widely in all walks of life. Currently, many companies are still reading the relevant information from the teaching device to monitor the robot state. Asthis kind of monitoring method needs technical personnel to record, a lot of technical personnel is wasted. Thus, development of remote monitoring system robot is imperative to solve this problem; and how to acquire the robot operation information locally is the key technology. At present, the monitoring system development technology mainly adopt $\operatorname{PLC}^{[1,2,3]}$, communication protocol ${ }^{[4,5,6,7,8]}$ (establish the communication between the Controller and PC), or the industrial Internet of Things technology [9] through the external sensor to obtain the robot state information. Despite the easy application, strong interface and good anti-jamming ability, PLC still have its limitations such as less information can be acquired (4-8)Using communication protocols to achieve the acquisition of robot state information always needs its own design,which increased the difficulty of monitoring

This paper is supported by

the key technologies R\&D program of Tianjin(18YFZCSF00600). system development. XIE, et al first adopted the industrial Internet of things technology to get robot state information, however, this method need to use external sensors to assist, which lead to a higher design cost. Compared with the above 3 methods, the $\mathrm{ABB}$ brand of industrial robots might be the better candidates to realize remote monitor due to its advantages of low development difficulty without designing communication protocols, high development efficiency and cost savings of system development. At present, foreign scholars have used the PC SDK to verify the high-frequency communication algorithm ${ }^{[10]}$, reducing the controller delay ${ }^{[11]}$.However, the previous study did not introduce the PC SDK clearly, so this paper gives a detailed description about the function of PC SDK in the first section. Furthermore, this paper puts forward a new set of $\mathrm{ABB}$ robot local multi-machine monitoring system combining with the PC SDK. Lastly, the paper defines the feasibility of this new monitoring system.

\section{PC SDK}

The PC SDK is a two-time development interface (API) reserved by $\mathrm{ABB}$ Industrial robots and is available. NET language call dynamic link library, through the API can establish the robot controller and PC-side application of Ethernet communication between the PC-side robot information reading and automatic mode of data modification, program control and other functions.

\section{A. Development environment and development mode}

The PC SDK has two kinds of development methods under virtual environment and real environment, in the virtual environment, it is necessary to connect the virtual controller established by Robotstudio simulation software to develop and debug the application, and the real environment needs to connect the true controller. Compared with the two development methods, the development method in the virtual environment is more convenient, the PC SDK can realize the communication between the virtual controller and the PC application, and the real environment needs the Ethernet communication with the real controller with the PC interface 
Communication module installed. Regardless of the development method, the PC SDK version used in PC applications must be compatible with the Robotware version used by the controller. The two development environment configurations are shown in Figure 1:

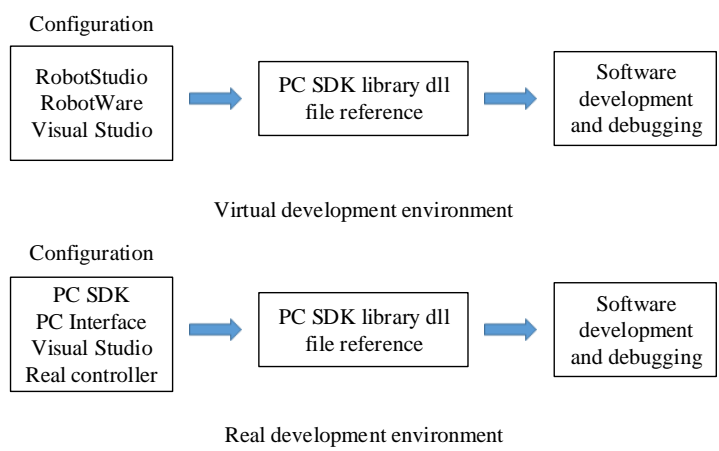

Fig. 1. Two development methods

\section{B. PC SDK class library composition and implementation functions}

The PC SDK class libraries are organized in the following domains:

1) Controllers

The classes used to access robot controller functionality together make up the Controller API (CAPI).

2) ConfigurationDomain

The Configuration domain namespace enables access to the configuration database of the controller. Using this domain you can read or write the value of a configuration parameter to the configuration database of the controller.

3) Discovery

To create a connection to the controller from a PC SDK application it has to make use of the Netscan functionality of the Discovery namespace. A NetworkScanner object must be created and a scan call must be performed.

4) EventLogDomain

Event log messages may contain information about controller status, RAPID execution, the running processes of the controller, and so on.

\section{5) FileSystemDomain}

The File System Domain namespace enables to create, save, load, rename, and delete files in the controller file system. It is also possible to create and delete directories.

\section{6) Hosting}

This is the main entrypoint for a host into the CAPI internal functions. It can be used to extend and alter the inner workings of the API.

7) IOSystemDomain

IO System Domain is a robot system uses input and output signals to control processes. Signals can be of digital, analog, or group signal type. Such I/O signals are accessible using the SDK.

\section{8) Messaging}

The Messaging domain of the PC SDK can be used to send and receive data between a PC SDK application and a RAPID task.

\section{9) MotionDomain}

The MotionDomain namespace lets you access the mechanical units of the robot system.

10) RapidDomain

The Rapid Domain namespace enables access to RAPID data in the robot system. There are numerous PC SDK classes representing the different RAPID data types. There is also a UserDefined class used for referring to the RECORD structures in RAPID.

11) UserAuthorizationManagement

In the robot controller there is a system controlling user access: the User Authorization System(UAS). If this feature is used each user needs a user name and a password to log on to a robot controller via RobotStudio. If the controller connection for any reason is lost, you need to log on again.

\section{ESTABLISH COMMUNICATION}

Using the development mode of the virtual environment to develop the monitoring application, the virtual workstation of IRB120 robot is built by using Robotstudio 5.15 simulation software to simulate the real robot working scene, and the virtual controller and PC communication can be realized. The IRB120 robot virtual workstation is shown in Figure 2: The workstation is able to control the robot with the $\mathrm{I} / \mathrm{O}$ signal to carry out the conveyor.

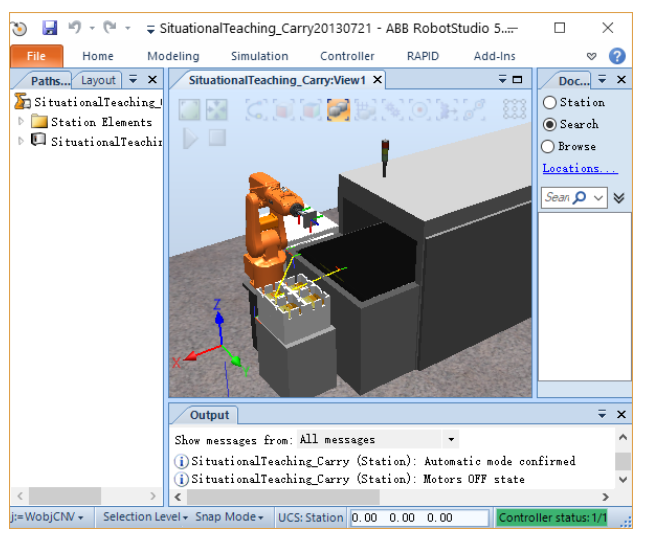

Fig. 2. IRB120 Robot Virtual workstation

Because the virtual controller is Robotware 5.15, create a new application under the X86 platform. NET Framework2.0 Framework, add a DLL file that references PC SDK 5.15, add a namespace, and complete the communication between the virtual controller and the PC. and using the Networkscanner class to monitor the controller on the communication network to test whether the virtual controller and PC-side communication is established successfully. Figure 3:pc SDK 5.15 dll file, Figure 4:pc SDK 5.15 version of the PC development platform diagram. 
ABB.PCSdk.Install.dll

- ABB.Robotics.Adapters.IRC5.dll

Q ABB.Robotics.Controllers.Configuration.dll

ABB.Robotics.Controllers.dll

ABB.Robotics.dll

Fig. 3. DLL file

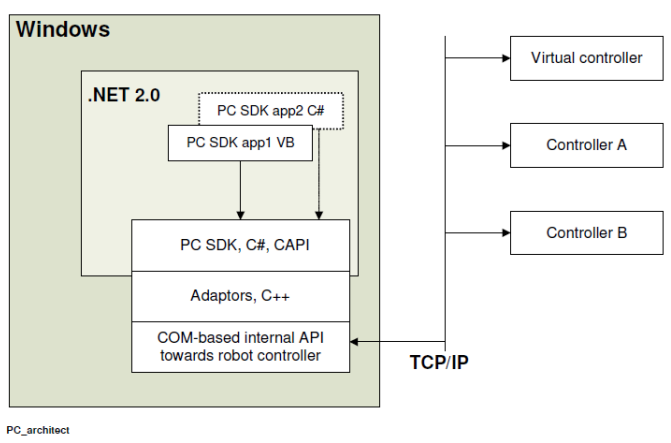

Fig. 4. PC development Platform

\section{MULTI-ROBOT MONITORING SYSTEM}

Based on the PC SDK in the virtual development environment, this paper develops a local multi-machine monitoring system for ABB IRB120 models, and the initial interface of the system is shown in Figure 5:

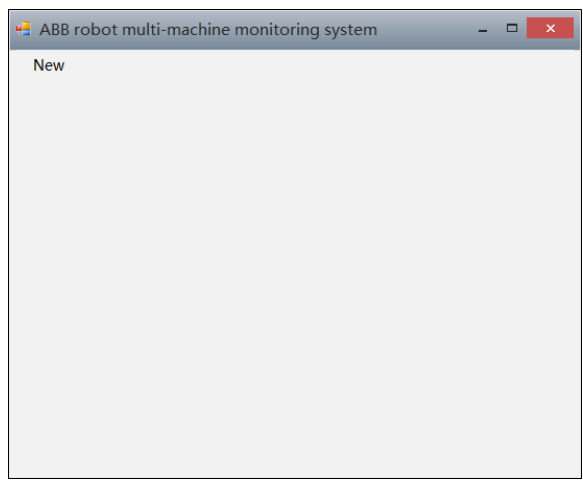

Fig. 5. Initial interface

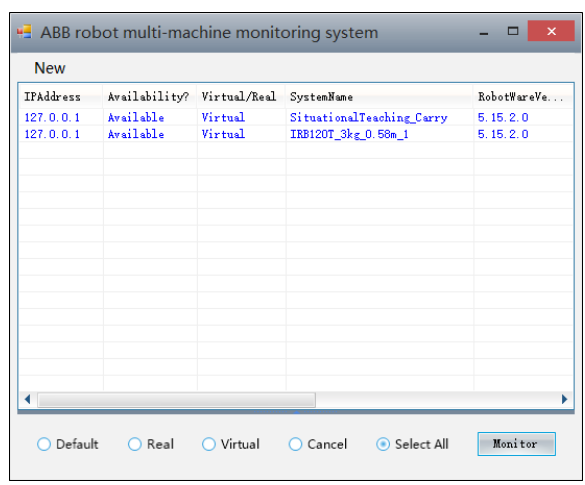

Fig. 6. Network scan Results
The multi-machine monitoring system includes the following functions:

1) Scan all robot controller information on the same network. As shown in Figure 6:

2) Select the robot to be monitored according to the controller information in the scan result and create a monitoring window.

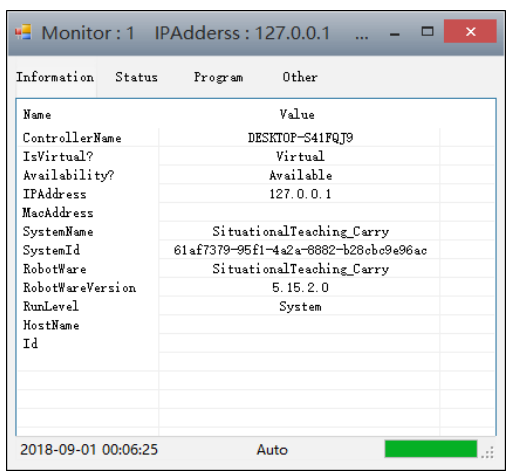

Fig. 7. Monitoring window 1

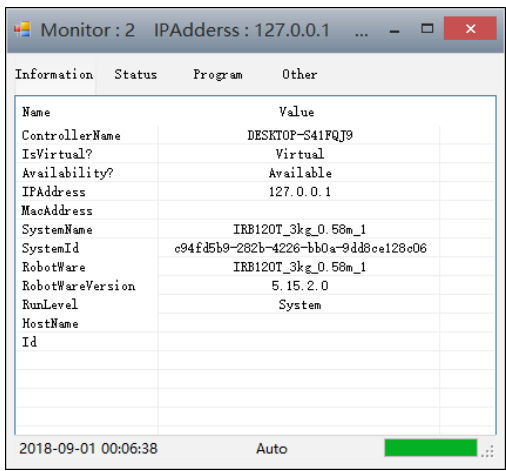

Fig. 8. Monitoring window 2

3) Obtain the monitored robot information, such as IPAddress, System name, robotware version number, etc., as shown in Figure 7, 8.

4) Always get the working state of the robot, such as the coordinate value of the TCP point, the rotation angle of the six axes, the I/O input status. As shown in Figure 9:

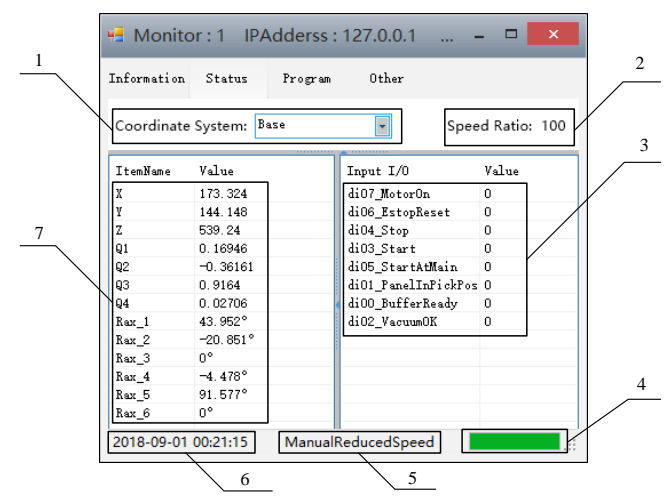

Fig. 9. Running status information 


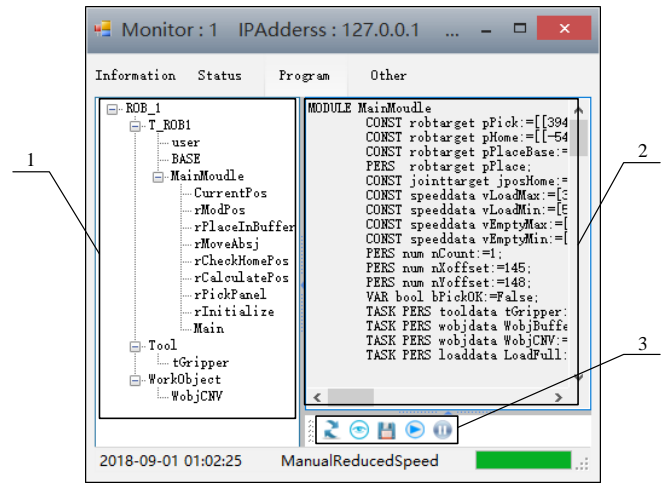

Fig. 10. Program page

The function in Figure 9 is described as:

1: Switching between the world coordinate system, the base calibration system, the workpiece coordinate system, the tool coordinate system, and the custom coordinate system.

2: The running speed ratio of the robot.

3: Input signal status value of the robot workstation.

4: Connection status of the robot controller.

5: The operating mode of the robot.

6: Robot system time.

7: $\mathrm{X}, \mathrm{Y}, \mathrm{Z}$ are the coordinate values of TCP in different coordinate systems, Q1, Q2, Q3, Q4 are the direction quantities of the robot in different coordinate systems, and Rax_1, Rax_2, Rax_3, Rax_4, Rax_5, Rax_6 are robots 1 to 6 -axis joint rotation angle value.

5) always acquire the mechanical unit, work task and routine of the current operating system of the robot, and view the text content of the routine program in the form of text, and can control the start and stop of the routine program. As shown in Figure 10:

The features in Figure 10 are:

1: Display all mechanical units, Task, Module, Routine, Tool, workobject information in the controller.

2: Show module text content.

3: Some operations on the routine. among them:

2 :Update the information in 1.

() :View routine text content.

$\Delta \quad$ :Save the module file from the controller to the local computer.

(-) :In automatic mode, start the routine program that is selected in 1.

(1) :Stop the 1 current routine program in automatic mode.

\section{MONITORING SYSTEM VERIFICATION}

The experimental verification of the multi-machine monitoring system was carried out using the established IRB120 robot virtual workstation (shown in Figure 5) in Section 3. The current pose of the robot in the virtual workstation is shown in Figure 11:

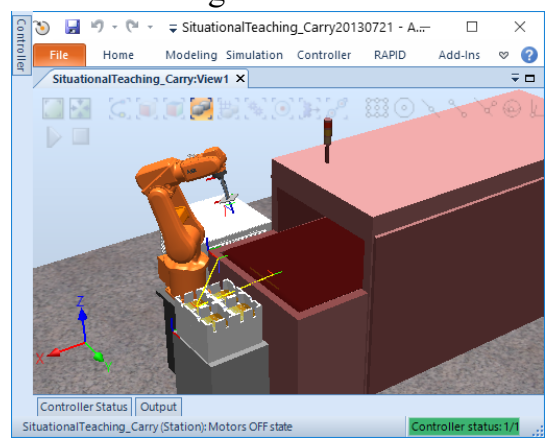

Fig. 11. Pose

The current TCP coordinate value and the direction vector value result of the robot, as shown in Figure 12 and 13:
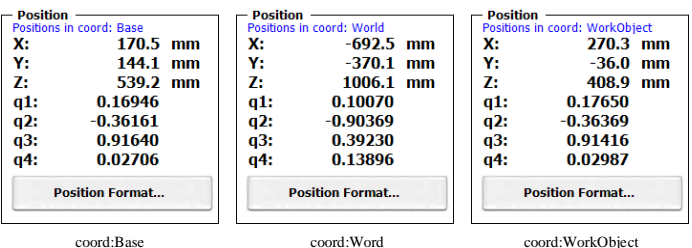

Fig. 12. TCP point coordinates and direction measures in the controller
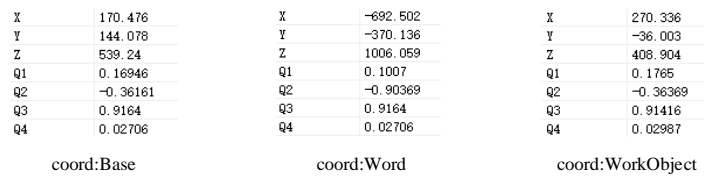

Fig. 13. TCP point coordinates and direction magnitude in the monitoring system

The current rotation angle value of the robot's 6 joints, as shown in Figure 14:
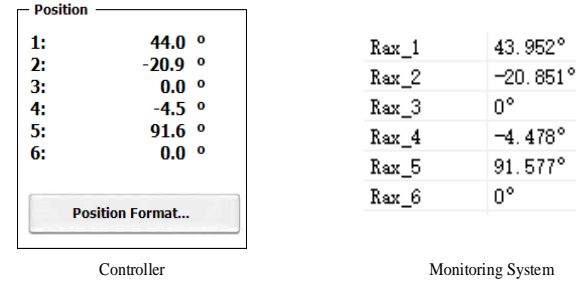

Fig. 14. Figure 141 to 6 axis joint rotation angle value

Query the current I/O system input signal value of the robot in the RoubotStudio simulation I/O module, as shown in Figure 15:

The multi-machine monitoring system monitors the current $\mathrm{I} / \mathrm{O}$ input signal in the robot status information as shown in Figure 16: 


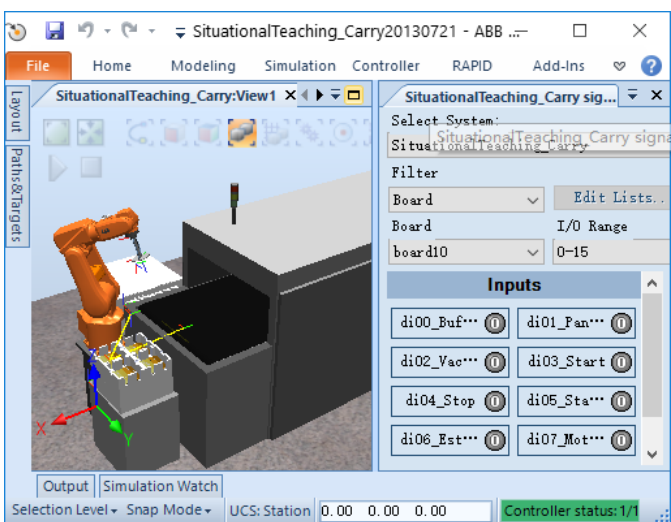

Fig. 15. Control system signal

\begin{tabular}{ll} 
Input I/0 & Value \\
di07_Motor0n & 0 \\
\hline di06_EstopReset & 0 \\
\hline di04_Stop & 0 \\
\hline di03_Start & 0 \\
\hline di05_StarthtMain & 0 \\
\hline di01_PanelInPickPos & 0 \\
\hline di00_BufferReady & 0 \\
\hline di02_Yacuum0K & 0 \\
\hline
\end{tabular}

Fig. 16. Monitoring system signal

The robot routine is queried in the controller, and the result is shown in Figure 17:

Multi-machine Monitoring System Monitor the current robot routine information as shown in Figure 18:

\begin{tabular}{|c|c|c|c|}
\hline ABD ig & $\begin{array}{l}\begin{array}{l}\text { Manual } \\
\text { SituationalTea... (DESKTOP-S4..) }\end{array} \\
\end{array}$ & $\begin{array}{l}\text { Guard Stop } \\
\text { Stopped (Speed 100\%) }\end{array}$ & $x$ \\
\hline \multicolumn{4}{|l|}{ T_ROB1/MainMoudle } \\
\hline \multicolumn{2}{|l|}{ Routines } & \multicolumn{2}{|l|}{ Active filter: } \\
\hline Name 1 & Module & Type & \\
\hline CurrentPos(... & MainMoudle & \multicolumn{2}{|l|}{ Function } \\
\hline Main() & MainMoudle & \multicolumn{2}{|l|}{ Procedure } \\
\hline rCalculatePos() & MainMoudle & \multicolumn{2}{|l|}{ Procedure } \\
\hline rCheckHomePos() & MainMoudle & \multicolumn{2}{|l|}{ Procedure } \\
\hline rInitialize() & MainMoudle & \multicolumn{2}{|l|}{ Procedure } \\
\hline rModPos() & MainMoudle & \multicolumn{2}{|l|}{ Procedure } \\
\hline rMoveAbsj() & MainMoudle & \multicolumn{2}{|l|}{ Procedure } \\
\hline rPickPanel() & MainMoudle & \multicolumn{2}{|l|}{ Procedure } \\
\hline File $\wedge$ & हु & Show Routine & \\
\hline 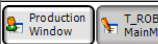 & & & RoB_1 \\
\hline
\end{tabular}

Fig. 17. Routine in the controller

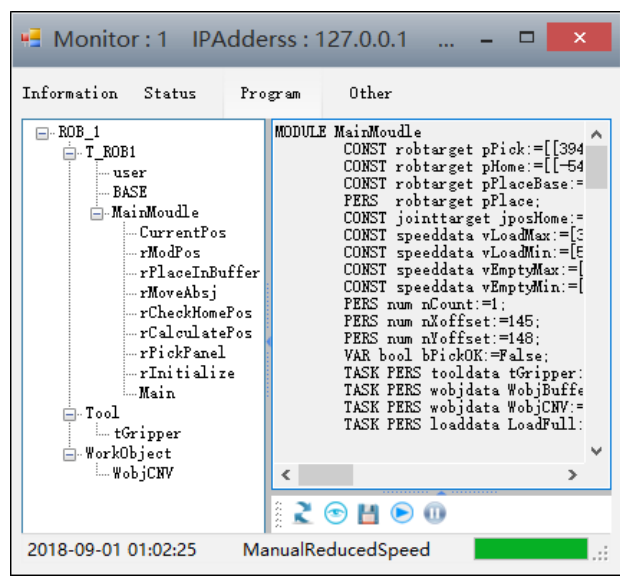

Fig. 18. Program monitoring results

\section{CONCLUSIONS}

The practicability of the PC SDK secondary development interface reserved by control system of ABB IRB120 robot based on the .Net platform was confirmed in this study. And a set of local multi-machine monitoring system for IRB120 industrial robot is designed.This system realized the local monitoring of IRB120 robot, and lays a practical foundation for the development of $\mathrm{ABB}$ Industrial robot remote Monitoring system in the future.

\section{REFERENCES}

[1] Wang Shengfa, Pan Min. Application of Communication between PLC and PC under VB in Industrial Robot Monitoring System[J]. Mechanical \& Electrical Engineering, 2001(06): 35-37.

[2] Wang Shengfa, Zhang Hongbing. Application of PLC Communication under VB in Industrial Robot Monitoring System[J]. Gansu Science and Technology, 2002(Z1): 55-56.

[3] Wang Yudong. Design of OPC-based industrial robot monitoring system and its arm fatigue life prediction [D]. Shanghai University of Engineering and Technology, 2016.

[4] Liu Lei. Design and development of industrial robot remote monitoring and diagnosis service system [D]. Dalian University of Technology, 2014.

[5] Chen Jie. Based on HRT-5 industrial robot network monitoring software development [D]. Wuhan Textile University, 2016.

[6] Pan Xiangfei. Research on industrial robot monitoring system based on Socket communication [D]. Zhejiang University of Technology, 2017.

[7] Lü Hantai. Research on Web3D Monitoring System for Industrial Robots [D]. Zhejiang University of Technology, 2017.

[8] Deng Sihao. Research and implementation of real-time robot monitoring and communication system [D]. Wuhan University of Technology, 2003.

[9] Wen Xiao. Research on robot testing system and remote monitoring platform based on industrial internet of things [D]. Anhui University of Engineering, 2016.

[10] Dalvand M M, Nahavandi S. Teleoperation of ABB industrial robots[J]. Industrial Robot-an International Journal, 2014, 41(3): 286-295.

[11] Dalvand M M, Nahavandi S. Improvements in teleoperation of industrial robots without low-level access[C]. systems, man and cybernetics, 2014: 2170-2175. 